\title{
The motion of particles in coaxially-arranged cylinders
}

\author{
Yu. M. Isaev*, V. F. Nekrashevich, N. M. Semashkin, and V. A. Zlobin \\ Ulyanovsk State Agrarian University, Ulyanovsk, Russia
}

\begin{abstract}
The purpose of the study outlined in the paper is to substantiate the technological and technical characteristics of the processes of moving a bulk material particle in the vertical direction. To determine the motion of the entire mass of material with a spiral, we used the simulation of steadystate particle motion using the d'Alembert equations in a cylindrical coordinate system.
\end{abstract}

\section{Introduction}

When calculating and designing vertically arranged spiral screw units, there is a need to use data that provide the relationship between the available parameters and the kinetics of motion of the entire flow of bulk material and its particles. A separate consideration deserves the case when the motion of the particle becomes constant (steady), and it moves along the inner surface of the cylindrical casing of the unit in the axial direction. Therefore, it is necessary to determine connections suitable for practical use in a particular spiral screw unit.

\section{Materials and methods of research}

We consider the case of a vertical arrangement of the unit, consisting of a spiral screw working body and two cylindrical coaxial housings (Fig. 1). The housings are fixedly mounted, and the spiral rotates between them around its axis with an unchanged angular velocity $\omega_{0}$.

For the fixed coordinates, we take the right-handed Cartesian coordinate system Oxyz, where $O z$ coincides with the vertical axis of the spiral. Suppose that the material point lies on the surface of the spiral, which is located between the coaxially mounted cylinders, while the point rotates around the axis with the angular velocity $\omega$ (Fig. 1).

During the movement of the material point by a spiral screw inside the coaxial cylinders, friction forces arise on the inner surface of the outer cylinder $F_{2}$ and the surface of the spiral $\mathrm{F}_{1}$. Figure 1 shows the following notation: $\mathrm{G}$ - gravity, $\mathrm{H} ; N_{2}$ is the force acting on the material point from the side of the inner surface of the outer cylinder, $\mathrm{H} ; N_{1}$ is the force acting on the material point from the side of the surface of the spiral, while it makes an angle $\theta$ with the normal to the helical line of the spiral, and the normal in turn is the angle $\alpha$ with the axis $\mathrm{Oz}$.

\footnotetext{
*Corresponding author: isurmi@yandex.ru
} 


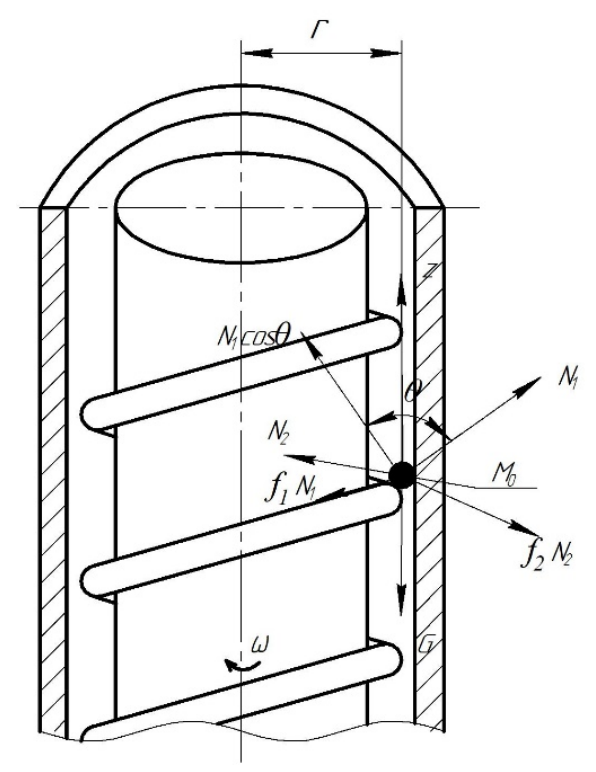

Fig. 1. Decomposition of force vectors applied to a material particle.

With the vertical movement, the material point moves in the following directions: 1 ) absolute, along a helical path, along the inner surface of the outer cylinder; 2) carrying together with a spiral turn; 3) relative - along a spiral turn [1, 2, 3, 4].

Let us consider in detail the forces acting on the material point (Fig. 2). Where $v_{0}$ is the velocity directed along the spiral; $v_{n}$ is the velocity vector of the carrying motion on a plane perpendicular to the axis of the spiral; $v$ is the absolute velocity vector. The axial velocity $v_{1}$ has a significant effect on the feed of a spiral screw unit and from Figure 2 one can see that it is defined as $v_{1}=v \sin \beta=v_{0} \sin \alpha$.

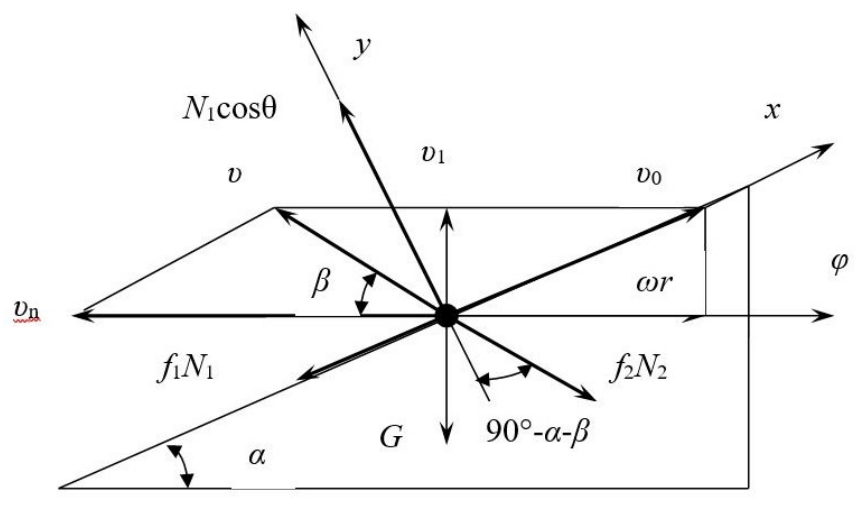

Fig. 2. Position of the forces acting on the material point on the developed view of the spiral.

If we place the vector $v_{0}$ on the plane perpendicular to the axis of the spiral, then we get the vectors $r \omega$ and $v_{1}$ which are directed at right angles to each other (Fig. 2), therefore, the expression for velocity is written:

$$
v_{1}=r \omega \tan \alpha,
$$

where $r$ is the radius of the inner surface of the outer cylinder $[5,6,7]$. 
This explains that the values of $v_{1}$ are determined by the angular velocity $\omega$. With the steady flow of bulk material, the equilibrium condition must be met

$$
\sum y=0, \quad \sum x=0 .
$$

Having projected the forces acting on the material point along the axes $Y$ and $X[8,9,10$, 11, 12, 13], we find:

$$
\begin{gathered}
N_{1} \cos \theta-G \cos \alpha- \\
-f_{2}\left(\omega-\omega^{\prime}\right)^{2} r \frac{G}{g} \sin (\alpha+\beta)=0 \\
-f_{1} N_{1}-G \sin \alpha-f_{2}\left(\omega-\omega^{\prime}\right)^{2} \times \\
\times r \frac{G}{g} \cos (\alpha+\beta)=0
\end{gathered}
$$

where $f_{1}$ - coefficient of friction of a particle on a spiral surface; $f_{2}$ - particle friction coefficient on the inner surface of the cylinder.

We multiply the equation (1) by $f_{2}$, and add it to (2) multiplied by $\cos \theta$. We reduce the resulting equation by $\mathrm{G}$ and multiply by minus one, we get:

$$
\begin{gathered}
f_{1} \cos \alpha+\cos \theta \sin \alpha+\frac{f_{2}\left(\omega_{0}-\omega\right)^{2}}{g} \times \\
\times r\left[f_{1} \sin (\alpha+\beta)-\cos \theta \cos (\alpha+\beta)\right]=0
\end{gathered} .
$$

From Fig. 2 we will find the trigonometric functions of the angle $\beta$ :

$$
\begin{gathered}
\sin \beta=\frac{v_{1}}{v}=\frac{v_{0} \sin \alpha}{\sqrt{\left(v_{0} \sin \alpha\right)^{2}+\left(v_{n}-v_{0} \cos \alpha\right)^{2}}} \\
=\frac{\cos \beta=\frac{v_{n}-v_{0} \cos \alpha}{v}=}{\sqrt{\left(v_{0} \sin \alpha\right)^{2}+\left(v_{n}-v_{0} \cos \alpha\right)^{2}}}
\end{gathered}
$$

We transform in these equalities the expression under the root:

$$
\begin{gathered}
\left(v_{0} \sin \alpha\right)^{2}+\left(v_{n}-v_{0} \cos \alpha\right)^{2}= \\
=v_{0}^{2}+v_{n}^{2}-2 v_{n} \cdot v_{0} \cdot \cos \alpha\left(v_{n}-v_{0} \cos \alpha\right)^{2} .
\end{gathered}
$$

Substituting in (4) and (5) the values of linear velocity $v_{0}=\omega \cdot r / \cos \alpha$ and $v_{n}=\omega_{0} \cdot r$ in terms of the angular velocity. Having denoted $k=\omega / \omega_{0}$ we obtain:

$$
\begin{aligned}
& \sin \beta=\frac{k \cdot \sin \alpha}{\sqrt{\cos ^{2} \alpha+k^{2}-2 k \cdot \cos ^{2} \alpha}} \\
& \cos \beta=\frac{(1-k) \cdot \cos \alpha}{\sqrt{\cos ^{2} \alpha+k^{2}-2 k \cdot \cos ^{2} \alpha}}
\end{aligned}
$$


By putting (7) and (8) in the expression (3) in square brackets and making trigonometric transformations, we obtain:

$$
\begin{gathered}
f_{1} \sin (\alpha+\beta)-\cos \theta \cos (\alpha+\beta)= \\
=\frac{f_{1} \cos \alpha \sin \alpha-\cos ^{2} \alpha \cos \theta+k \cos \theta}{\sqrt{\cos ^{2} \alpha+k^{2}-2 k \cdot \cos ^{2} \alpha}} .
\end{gathered}
$$

In view of the obtained dependences we will write the relation (3) in the form:

$$
\begin{gathered}
\frac{f_{2} r \omega_{0}^{2}(1-k)^{2}\left(f_{1} \cos \alpha \sin \alpha-\cos ^{2} \alpha \cos \theta+k \cos \theta\right)}{\sqrt{\cos ^{2} \alpha+k^{2}-2 k \cdot \cos ^{2} \alpha}} \\
=-f_{1} \cos \alpha+\cos \theta \sin \alpha
\end{gathered}
$$

We introduce in our consideration the criterion $\lambda=r \omega_{0}^{2} / g$ which characterizes the kinematic mode of the helical screw. Having solved the equation (10) relative to the coefficient $\lambda$, we obtain the equation an equation for determining the particle velocity depending on the parameters of a spiral screw unit:

$$
\begin{gathered}
\lambda=\frac{r \omega_{0}^{2}}{g}=\frac{\left(f_{1} \cos \alpha+\cos \theta \sin \alpha\right)(1-k)^{2}}{f_{2}(1-k)^{2}} \times \\
\times \frac{\sqrt{\cos ^{2} \alpha+k^{2}-2 k \cdot \cos ^{2} \alpha}}{\left(\cos ^{2} \alpha \cos \theta-f_{1} \cos \alpha \sin \alpha-k \cos \theta\right)}
\end{gathered}
$$

The sought root $k$ of the equation (11) cannot be imaginary or negative and must have a real and positive value. The possibility of a particle transfer is a consequence of the existence of centrifugal force $N=\left(\omega_{0}-\omega\right)^{2} \cdot r \cdot G / g$ and friction force $F=f_{2} \cdot N$. The relation $k=\omega / \omega_{0}$ is the proper fraction, since at $k=\omega / \omega_{0}=0$ it follows that $\omega=0$ - this means that a particle is not moved along the helical line and if $\omega=\omega_{0}$, then $k=1$ and $\sin \beta$ will be equal to

$$
\sin \beta=\frac{k \cdot \sin \alpha}{\sqrt{\cos ^{2} \alpha+k^{2}-2 k \cdot \cos ^{2} \alpha}}=1 \cdot \frac{\sin \alpha}{\sqrt{1-\cos ^{2} \alpha}}=1,
$$

from which $\beta=90^{\circ}$, that is the vector $v$ lies vertically but this is impossible because there are no forces that can lift a particle:

$$
N=\left(\omega_{0}-\omega\right)^{2} \cdot r \cdot G / g=0
$$

Solving the equation (11) for each specific case with definite values of $f_{1}$ and $f_{2}$, one can choose spiral screw units with the greatest capacity and axial velocity $v_{1}=\omega \cdot r \tan \alpha$ or when the quantity has the maximum value

$$
\frac{v_{1}}{v_{n}}=\frac{\omega \cdot r \cdot \tan \alpha}{\omega_{0} \cdot r}=k \cdot \tan \alpha .
$$


If we take the friction coefficient of a material particle for steel $f_{1}=f_{2}=0.5$ and plot the graphs of $k$ in the function $\lambda=r \omega_{0}^{2} / g$ of the equation (11) for $\alpha=15^{\circ}, 20^{\circ}, 25^{\circ}, 30^{\circ}$ which are given in Fig. 3.

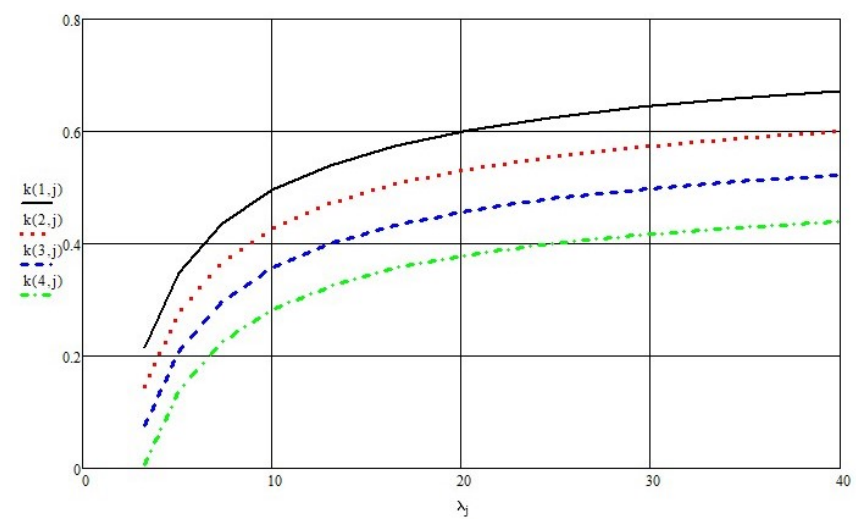

Fig. 3. Changes $k=\omega / \omega_{0}$ depending on $\lambda=r \omega_{0}^{2} / g$ with the selected characteristics of the spiral unit for various values of the inclination angle of the helical line of the spiral. Notation: $\mathrm{k}(1, \mathrm{j})-\alpha=15^{\circ} ; \mathrm{k}(2, \mathrm{j})-\alpha=20^{\circ} ; \mathrm{k}(3, \mathrm{j})-\alpha=25^{\circ} ; \mathrm{k}(4, \mathrm{j})-\alpha=30^{\circ}$.

Let us find out the condition under which the particle will not be lifted up. Putting in the equation (3) $\beta=0^{\circ}, \cos \beta=1, \sin \beta=0$, we have:

$$
\begin{gathered}
f_{1} \cos \alpha+\cos \theta \sin \alpha+\frac{f_{2}\left(\omega_{0}-\omega\right)^{2}}{g} \times \\
\times r\left[f_{1} \sin \alpha-\cos \theta \cos \alpha\right]=0
\end{gathered}
$$

Next, we will obtain

$$
\omega_{0}-\omega=\sqrt{\frac{\left(f_{1} \cos \alpha+\sin \alpha \cos \theta\right) \cdot g}{r f_{2}\left(\cos \alpha \cos \theta-f_{1} \sin \alpha\right)}},
$$

or

$$
\omega_{0}=\omega+\sqrt{\frac{\operatorname{tg}\left(\alpha+\varphi_{1}\right) \cdot g}{r f_{2}}},
$$

where $\varphi_{1}=\operatorname{arctg}\left(\frac{f_{1}}{\cos \theta}\right)$.

Consequently, for the motion of the mass up along the auger axis it is necessary to satisfy the condition

$$
\omega_{0} \geq \sqrt{\frac{\operatorname{tg}\left(\alpha+\varphi_{1}\right) \cdot g}{r f_{2}}} .
$$

We solve the problem of the axial velocity of the material in a vertical spiral screw unit. 


\section{Results of the research}

The equation (12) describes the relative motion of a particle in a vertical spiral unit.

Figure 3 shows the results of calculations of the axial velocity of the spiral and particle movement in a spiral screw unit with the following characteristics; $r=0.02 \mathrm{~m}$ is the inner radius of the outer cylinder; $d=0.004 \mathrm{~m}$ is the diameter of the spiral bar; $r_{1}=0.004 \mathrm{~m}$ is the average radius of the particle of the moved material; $r_{2}=0.018 \mathrm{~m}$ is the radius of the spiral.

The obtained equation and dependencies allow one to choose the optimal parameters in the calculation and design of spiral-helical conveyors.

The experimental studies were carried out on a unit for conveying soybeans with the use of a spiral screw working body located between vertically arranged coaxial cylinders.

Figure 4 shows the results of the experimental studies on the movement of soybeans: spiral screw working bodies with an outer diameter of $d=33 \mathrm{~mm}$, a helix pitch of $S=25$ $\mathrm{mm}$, a wire diameter of $4 \mathrm{~mm}$, a cylinder length of $L=1 \mathrm{~m}$, an inner diameter of the outer cylinder $D_{1}=36.5 \mathrm{~mm}$, the outer diameter of the inner cylinder $D_{2}=21 \mathrm{~mm}$, the angular velocity of rotation of the spiral in this case varied from $\omega=40$ to $\omega=88 \mathrm{~s}^{-1}$. The transferred material is soybeans with bulk density $\rho=750 \mathrm{~kg} / \mathrm{m}^{3}$.

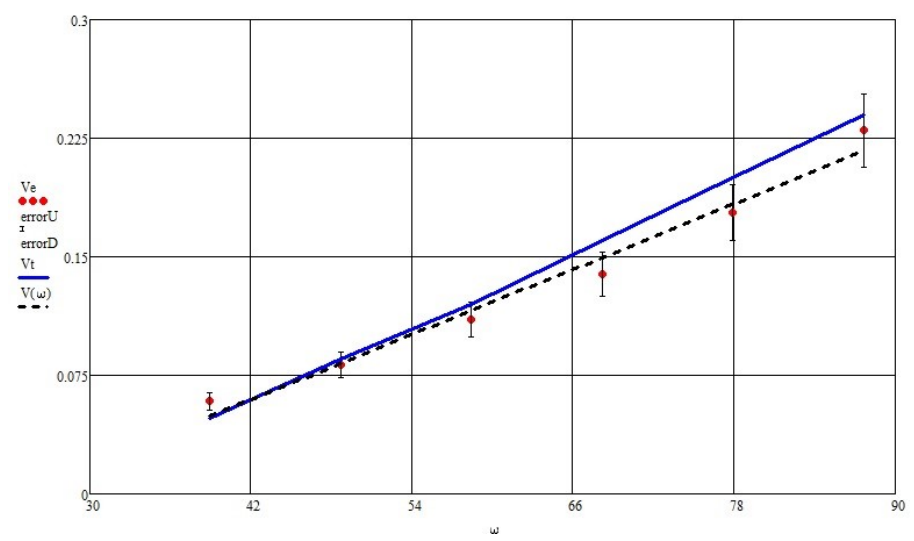

Fig. 4. The graph of the axial velocity $V, \mathrm{~m} / \mathrm{s}$ of soybeans versus the angular velocity of the spiral rotation $\omega, \mathrm{s}^{-1}$.

Notation: Ve - experimental relationship; Vt - theoretical relationship; V( $\omega)$ - regression.

The analysis of Figure 4 shows that the experimental values of the axial velocities of individual particles versus the frequency of rotation of the spiral are consistent with theoretical results. As a result of processing the experimental data, a regression equation is obtained for the dependence of the axial velocity $V(\omega), \mathrm{m} / \mathrm{s}$ of the movement of soybeans on the angular velocity of rotation of the spiral screw $\omega, \mathrm{s}^{-1}$ :

$$
V(\omega)=3,4 \cdot 10^{-3} \omega-0,085
$$

\section{Conclusions}

From this formula, one can determine the threshold critical value of the angular velocity of the spiral rotation, at which the particle begins to move in a vertical coaxial cylindrical casing. For experimental research conditions this is critical $\omega_{\text {crit }}=25 \mathrm{~s}^{-1}$.

The work was carried out in the framework of a grant of the President of the Russian Federation for state support of young Russian scientists - candidates of sciences MK-3511.2019.8 


\section{References}

1. Kotov D.N, Isaev Yu.M, Kryuchin N.P, Semashkin N.M and Kryuchin A.N, Determination of Speed of Movement of The Particle on The Rotating Cone with Shovels, International Journal of Mechanical Engineering and Technology (IJMET)10(2), 2019, pp. 1507-1514. http://www.iaeme.com/ijmet/issues.asp?JType=IJMET\&VType=10\&IType=2.

2. Vasiliev, V.F. Water-jet propulsion / MADI (GTU). - M. - 2006. - p.45.

3. P.M. Konkov, Mechanization of grain unloading (Moscow, Kolos, 1972).

4. G.A Roginsky, Dosing of bulk materials (Moscow, Khimiya, 1978, p. 176).

5. G. O. Harries, Application of a radioizotope to the determination of the annular thicknesl of grain in an anger conveyor, Journal of Agricultural Engineering Research, 1, 1962.

6. L.O. Chernilov, The equipment of elevators and warehouses (Moscow, Kolos, 1977, p. 319).

7. L.D. Landau, E.M. Livshits, Theoretical Physics (Textbook. In 10 vols, T. VI. Hydrodynamics. -4 th ed., Sr. Moscow, 1988, p.736).

8. Zheltov, V.P. Calculation of spiral conveyors / V.P. Zheltov // Bulletin of machine building. - 1975. - No. 5. - pp. 18 ... 21.

9. L.A. Dorfman, Hydrodynamic resistance and heat transfer of rotating bodies (Moscow, Fizmatgiz, 1960, p. 260).

10. G.I. Kogan-Wolman, Flexible wire shafts (Moscow, Mashgiz, 1957, p. 247).

11. L.I. Sedov, Similarity and dimension methods in mechanics (Moscow, 1954, p. 328).

12. Isaev Yu. M., Semashkin N. M., Kalenkov S. A. Specification of particle movement in a spiral - screw element, Vestnik of Ulyanovsk state agricultural academy, 4(44), 2018, pp. 6-11. DOI 10.18286/1816-4501-2018-3-6-11.

13. Isaev Yu. M, Kryuchin N.P, Semashkin N. M and Kryuchin A. N, Theoretical Studies of Movement of Loose Material in a Dosing Device, International Journal of Mechanical Engineering and Technology, 9(5), 2018, pp. 834-840 http://www.iaeme.com/IJMET/issues.asp?JType=IJMET\&VType=9\&IType=5 